\title{
Transient Dynamics Measurement of Light-material Interaction for Laser Optics
}

\author{
Zhichao Liu ${ }^{1, *}$, Feng Geng ${ }^{1}$, Yaguo Li ${ }^{1}$, Qinghua Zhang ${ }^{1}$, Jian Wang ${ }^{1}$ and Qiao $\mathrm{Xu}^{1}$ \\ ${ }^{1}$ Fine Optics Engineering Research Center, Chengdu, 610041, China
}

\begin{abstract}
Laser damage performance is an important parameter to evaluate the quality of high power laser optical components. Understanding the physical mechanism of the interaction between laser and optical material is of great significance for improving the laser damage resistance of optical elements. For nanosecond pulsed laser, the time duration of laser-induced damage is ultra-short, and the measurement of related physical quantities is very difficult. Therefore, the Time Resolved Pump and Probe (TRPP) technique can effectively solve this problem. TRPP shadow imaging can obtain the transient dynamic information in range of picosecond to microsecond, including the plasma formation in the early stage, the shock wave propagation, the crack expanding, material ejection and other phenomena. Moreover, the ultra-fast physical quantities can be accurately measured by employing two detective beams with perpendicular polarization directions, for example $\mathrm{S}$ and $\mathrm{P}$ polarization light. All those information provides effective experimental data to reveal the physical mechanism of damage process.
\end{abstract}

\section{Introduction}

The interaction mechanism between high-power laser and optical material in nanosecond region is a quite important issue for various researches, such as laser assisted coating, laser ablation and laser induced damage. For general broadband transparent dielectric materials [1], the laser energy density required for inducing ionization breakdown usually exceeds to $10^{11} \mathrm{~W} / \mathrm{cm}^{2}$. The temperature of the irradiated area increases to $1 \mathrm{eV} \sim 10 \mathrm{eV}$ and the local pressure exceeds to $10 \mathrm{GPa} 100 \mathrm{GPa}$ [2] in an ultra-fast time. A variety of physical phenomena will be involved in this process, such as the shockwave propagation, crack expanding, stress conduction and light absorption evolution. They are important to reveal the interaction mechanism of laser and material in the early time. Characterization of the physical parameters in this region will be useful to build theoretical model and understand the mechanism of light-matter interaction.

The Time Resolved Pump and Probe (TRPP) technique can effectively solve this problem. TRPP shadow imaging can obtain the transient dynamic information in range from ps to ms. Moreover, the ultra-fast physical quantities can be accurately measured by employing two detective beams with perpendicular polarization directions. In this paper, we introduce the TRPP device and its freatures. The plasma formation in the early stage, shock wave propagation, crack expanding and material ejection are investigated as the typical application of TRPP shadow imging technique in transient measurement field.
The principle of TRPP technology is illuminated in Fig. 1. It contains pump laser, probe laser, digital delay device, optical delay line and shadow imaging system. The pump laser provides a nanosecond pulse to irradiate the sample surface and creates damage region. In the meanwhile, a picosecond pulse functioning as the probe laser goes through this region to take the transient image, grabbing a time-slice. Finally, the transient image is recorded and extracted by the shadow imaging system. The different time-slices of the whole damage event can be grabbed by shifting the delay between the pump and the probe pulse. In order to measure the transient parameter quantitatively, we need to grab two slices for one damage event. To achieve this purpose, the probe laser is split into two beams with orthogonal polarization directions, $\mathrm{S}$ light and $\mathrm{P}$ light, by use of a polarized beam splitter (see PBS in Fig. 1). The relative delay between them is controlled by an optical delay line, as denoted by the dotted line frame in Fig. 1. After that, the two beams are combined coaxially by another PBS and go through the damage region together. Passing the damage region, the beams are split to $\mathrm{S}$ and $\mathrm{P}$ lights again by the third PBS. Two microscopes are deployed to grab the transient images of S and P lights as two time-slices.

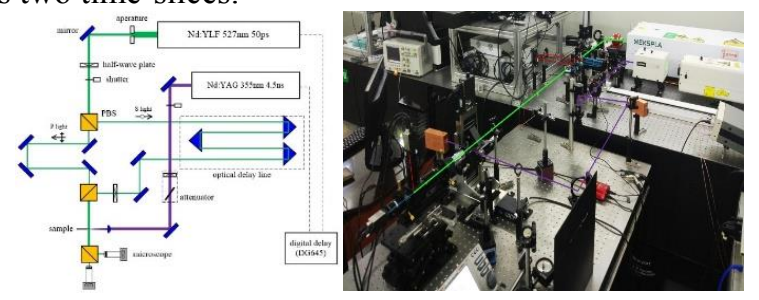

Fig.1 Schematic diagram of the TRPP setup.

\section{Principle and device}

\footnotetext{
Corresponding author: zcliu44@163.com
} 


\section{Results and discuss}

\subsection{Plasmas formation}

The avalanche ionization region continues expanding to explosion and then induces the shockwave. The transient images of this early stage were caught by the TRPP method shown in Fig. 2. The shadow cluster is the ionization phenomenon, for example, pointed by the white arrow in Fig. 2(b). Once the probe light is absorbed by plasma, the dark image will appear. Therefore, by measuring the onset time, position and size of shadow cluster, we can obtain the evolution of plasmas region.

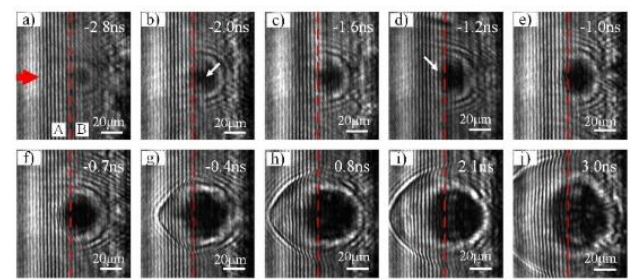

Fig. 2. The propagation of plasma and shockwave in pump laser pulse duration from $-2.8 \mathrm{~ns}$ to $3.0 \mathrm{~ns}$. Red dotted line means the interface of air side (signed as A) and bulk side (signed as B). Pump laser comes along with red arrow.

\subsection{Shockwave propagation}

The Fig. 2 shows the image series of shockwave propagation in the air side under nanosecond laser pumping. Each sub-image is caught with a certain delay. The pump laser comes along with the red arrow. By using $\mathrm{P} / \mathrm{S}$ light method, the velocity of shock wave can be achieved as shown in Fig. 3. Its decay rule obeys the Taylor-Sedov's point explosion model [3].

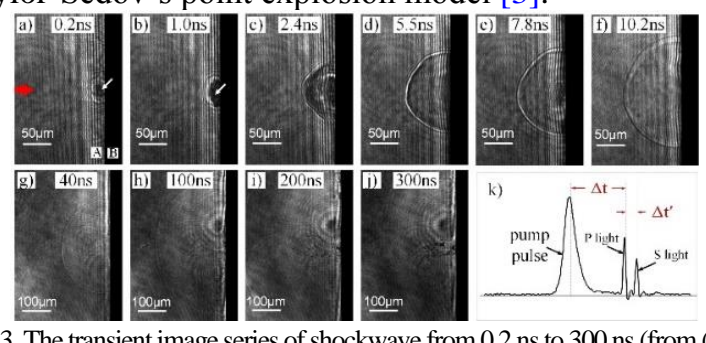

Fig. 3. The transient image series of shockwave from $0.2 \mathrm{~ns}$ to $300 \mathrm{~ns}$ (from (a) to (j)) and the schematic diagram of S-P light method (k). Pump laser comes along with red arrow and the white arrow means the plasma region.

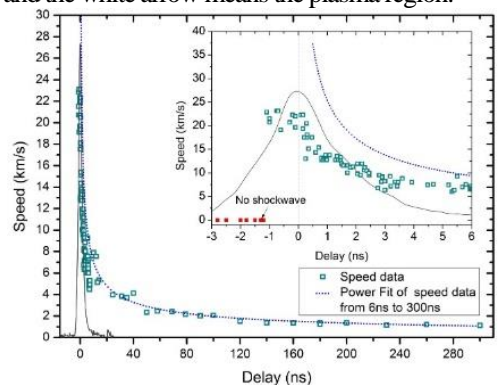

Fig. 4. The relationship between shockwave speed and delay from $-1.2 \mathrm{~ns}$ to $300 \mathrm{~ns}$ under $0.77 \mathrm{~mJ}$ pump energy. The blue dotted line is the fitting curve of shock wave speed along with delay. And the grey curve is the contour of pump laser duration.

\subsection{Crack expanding}

Use Vickers indenter to suppress artificial cracks on the surface of KDP crystal. The dynamic evolution of indentation pits and cracks towards damage under laser irradiation was observed, see Fig. 5. The upper images were taken under bright field and lower ones were under dark field. The results indicates a clear correlation between the position of plasma absorption cluster and the crater crack.

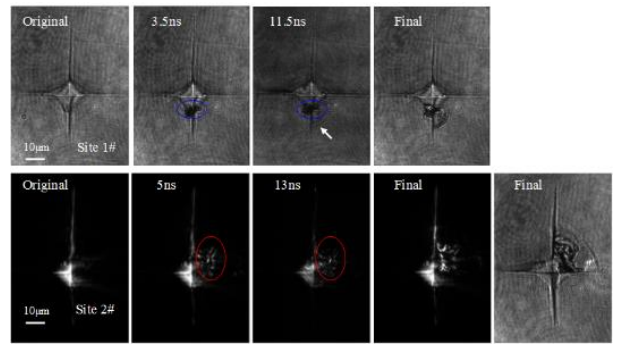

Fig. 5. Dynamics of the cracks expanding under laser irradiation

\subsection{Material ejection}

By using TRPP technology, the material ejection process can be observed, see Fig. 6. Some important physical quantities such as ejection angle, velocity and onset time can be obtained, which can provide experimental support for the establishing the mechanical fracture model.

-The ejection angle of the rear surface is larger than that of the front surface.

-Two stages: For delay less than $\sim 1 \mu \mathrm{s}$, melted and spherical particles. For delay more than $1 \mu \mathrm{s}$, irregular fragments with larger size and lower speed.
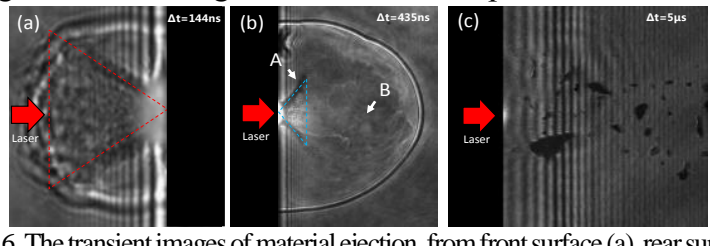

Fig. 6. The transient images of material ejection, from front surface (a), rear surface (b) and the debris ejection for longer delay.

\section{Conclusion}

In summary, the TRPP technique discrribed in this paper can be used to reveal the transient dynamics process of light-matter, espectically for high power laser-induced damge. Some most important physical parameters, such as plasmas size, shockwave speed and material ejection angle, could be measured quantitatively. Thanks to the TRPP technique, we can get the rule of ultra-fast process and to understand the mechanism of laser-induced damage.

\section{References}

1. B. C. Stuart, M. D. Feit, S. Herman, A. M. Rubenchik, B. W. Shore, and M. D. Perry, Phys. Rev. B 53, 1749 (1996)

2. Carr, C., Radousky, H., Rubenchik, A., Feit, M., and Demos, S., Phys. Rev. Lett. 92, 087401 (2004)

3. Geoffrey Taylor, Proc. Royal Sco. London, Ser. A201 (1950) 